\title{
Enzymatic degradation of in vitro Staphylococcus aureus biofilms supplemented with human plasma
}

This article was published in the following Dove Press journal:

Infection and Drug Resistance

27 April 2016

Number of times this article has been viewed

\section{Chase MWatters ${ }^{1,2}$ \\ Tarea Burton' \\ Dickson K Kirui' \\ Nancy J Millenbaugh'}

'Maxillofacial Injury and Disease Department, Naval Medical Research Unit San Antonio, Joint Base San Antonio-Fort Sam Houston, TX, USA; ${ }^{2}$ Wound Infections Department, Naval Medical Research Center, Silver Spring, MD, USA
Correspondence: Nancy J Millenbaugh Maxillofacial Injury and Disease Department, Naval Medical Research Unit San Antonio, 3650 Chambers Pass, Building 3610, Joint Base San Antonio-Fort Sam Houston, TX 78234-63 I5, USA

$\mathrm{Tel}+\mid 2105397133$

Fax + I 210539931 I

Email nancy.j.millenbaugh.civ@mail.mil
Abstract: Enzymatic debridement is a therapeutic strategy used clinically to remove necrotic tissue from wounds. Some of the enzymes utilized for debridement have been tested against bacterial pathogens, but the effectiveness of these agents in dispersing clinically relevant biofilms has not been fully characterized. Here, we developed an in vitro Staphylococcus aureus biofilm model that mimics wound-like conditions and employed this model to investigate the antibiofilm activity of four enzymatic compounds. Human plasma at concentrations of $0 \%-50 \%$ was supplemented into growth media and used to evaluate biofilm biomass accumulation over 24 hours and 48 hours in one methicillin-sensitive and five methicillin-resistant strains of $S$. aureus. Supplementation of media with $10 \%$ human plasma resulted in the most robust biofilms in all six strains. The enzymes $\alpha$-amylase, bromelain, lysostaphin, and papain were then tested against $S$. aureus biofilms cultured in 10\% human plasma. Quantification of biofilms after 2 hours and 24 hours of treatment using the crystal violet assay revealed that lysostaphin decreased biomass by up to $76 \%$, whereas $\alpha$-amylase, bromelain, and papain reduced biomass by up to $97 \%, 98 \%$, and $98 \%$, respectively. Scanning electron microscopy confirmed that the dispersal agents detached the biofilm exopolysaccharide matrix and bacteria from the growth surface. Lysostaphin caused less visible dispersal of the biofilms, but unlike the other enzymes, induced morphological changes indicative of bacterial cell damage. Overall, our results indicate that use of enzymes may be an effective means of eradicating biofilms and a promising strategy to improve treatment of multidrug-resistant bacterial infections.

Keywords: MRSA, $\alpha$-amylase, bromelain, lysostaphin, papain

\section{Introduction}

Staphylococcus aureus is a leading cause of infection in military and civilian populations throughout the world. Wounds infected with $S$. aureus are especially difficult to treat due to the prevalence of multiple drug-resistant strains and proclivity for biofilm formation in this pathogen. Bacterial biofilms are complex, often multispecies microbial communities composed of cells that are enclosed in a self-produced exopolysaccharide (EPS) matrix and attached to a surface or to each other. ${ }^{1}$ Biofilm formation confers multiple advantages for cell survival compared to planktonic growth, including protection from host immune defenses, shear stress, disinfectants, and antibiotics by the EPS matrix. ${ }^{1}$ The barrier properties of the biofilm matrix also create an anoxic and low nutrient environment that induces cellular dormancy associated with multidrug tolerance, a transient and nonheritable phenotype that differs from classic mechanisms of antibiotic resistance. ${ }^{1}$ The physically protected and antibiotic-tolerant population of cells within a biofilm is thought to act as a reservoir for recurrent infections in 
nonhealing wounds and dissemination of bacteria throughout the host. Therefore, use of novel therapies that specifically attack biofilm-associated infections, for example, by promoting biofilm dispersal ${ }^{2,3}$ or killing dormant persistor cells, ${ }^{4}$ is a promising strategy for significantly improving patient outcomes.

An essential component in the management of chronic wounds is the use of debridement to remove necrotic and infected tissue and promote healing. Numerous debridement techniques are employed clinically, including mechanical, surgical, osmotic, ultrasound, enzymatic, and maggot therapies. ${ }^{5}$ While mechanical and surgical methods are commonly preferred due to their rapidness and specificity, these procedures can be associated with pain, collateral tissue damage, extended hospital stays, and high cost. ${ }^{5}$ Enzymatic debridement is an attractive alternative for certain patients because it is less painful, can be applied at the bedside, and, notably, has potential effectiveness against a broad range of bacterial wound pathogens. ${ }^{5}$ Hence, the need for improved biofilm treatment strategies has renewed interest in developing enzymatic compounds for wound management, especially those that attack components of the EPS matrix.

The most commonly used and effective enzymatic debridement agents include papain/urea, bromelain, DNase I/fibrolysin, krillase, sutilains, and collagenase. ${ }^{6}$ Known mechanisms of action for many of these compounds indicate that, in addition to removing necrotic tissue, they may possess activity against proteins and bacterial or host DNA within the biofilm matrix. ${ }^{7}$ Although enzymes have been utilized for clinical debridement since the 1940s, there remains a gap in knowledge regarding their efficacy against biofilms formed by specific bacterial strains and the optimal treatment regimens required to effectively degrade the EPS matrix. Recent preclinical studies have investigated the ability of various enzymatic agents, for example, DNase $\mathrm{I},{ }^{7}$ dispersin $\mathrm{B},{ }^{7} \alpha$-amylase, ${ }^{8}$ lysostaphin, ${ }^{9}$ and proteinase $\mathrm{K},{ }^{7}$ to disperse or inhibit $S$. aureus biofilms. However, the in vitro models used in many of these studies were limited due to the type of bacterial strains selected for testing or because the culture conditions lacked key host factors that significantly affect biofilm formation. ${ }^{10}$ Thus, the focus of this study was to test enzymatic dispersal agents against $S$. aureus biofilms using an in vitro human plasma biofilm model and $S$. aureus clinical wound isolates. Enzymatic debridement agents that have either been used clinically or can easily be transitioned to the clinical setting were selected for this investigation. In addition, compounds with differing mechanisms of action, that is, proteases, an antipolysaccharide, and an antipeptidoglycan, were included in this study.

\section{Materials and methods}

\section{Bacterial strains}

The bacterial strains used in this study included methicillinsensitive $S$. aureus (ATCC 29213), methicillin-resistant S. aureus (MRSA; ATCC 33591), and four MRSA clinical wound isolates, namely, IQ0070, SA5214, SA5123, and SA5120. Bacterial stocks were maintained at $-80^{\circ} \mathrm{C}$. The Naval Medical Research Unit San Antonio Institutional Review Board determined that use of bacteria isolated from clinical specimens in this study is not human subjects research because the bacteria were obtained without identifiers, from strain repositories, were not collected specifically for the current research project through an interaction or intervention with living individuals, and the investigators cannot readily ascertain the identity of the patients from whom the bacterial strains were collected.

\section{Dispersal enzymes}

Enzymes were purchased from Sigma-Aldrich Co. (St Louis, MO, USA) and included $\alpha$-amylase from Bacillus subtilis (Cat No 10069, 380 U/mg), bromelain extracted from pineapple stem (Cat No B4882, $\geq 3 \mathrm{U} / \mathrm{mg}$ ), lysostaphin from Staphylococcus simulans (Cat No L9043, $\geq 3,000 \mathrm{U} / \mathrm{mg}$ ), and papain extracted from Carica papaya (Cat No 76220, $\geq 3 \mathrm{U} / \mathrm{mg}$ ). Stock solutions of lysostaphin were prepared in $20 \mathrm{mM}$ sodium acetate at $\mathrm{pH} 4.5$ and stored at $-20^{\circ} \mathrm{C}$. Working dilutions were prepared daily using $20 \mathrm{mM}$ Tris- $\mathrm{HCl}$ with $100 \mathrm{mM} \mathrm{NaCl}$ at $\mathrm{pH}$ 7.4. Working concentrations of the other enzymes were prepared daily from the powder form using $20 \mathrm{mM}$ Tris- $\mathrm{HCl}$ with $100 \mathrm{mM} \mathrm{NaCl}$ at $\mathrm{pH}$ 7.4.

\section{Biofilm treatment and crystal violet assay}

$S$. aureus was grown overnight in tryptic soy broth (TSB) at $37^{\circ} \mathrm{C}$ with shaking. To test the effect of human plasma on biofilm formation, the overnight cultures were diluted to an optical density $\left(\mathrm{OD}_{600}\right)$ of 0.1 using TSB supplemented with $0 \%, 1 \%, 10 \%$, or $50 \%$ human plasma. Bacterial suspensions were then added to 96 -well polystyrene plates, and biofilms were grown statically at $37^{\circ} \mathrm{C}$ for 24 hours. To perform the crystal violet $(\mathrm{CV})$ assay, biofilms were washed with phosphate-buffered saline (PBS), stained with $0.01 \% \mathrm{CV}$ for 30 minutes, and washed again with PBS. The CV dye was eluted using 95\% ethanol and quantified by measuring $\mathrm{OD}_{595}$ with a BioTek ${ }^{\circledR}$ Synergy HT microplate reader. Blank samples, which received TSB with the appropriate concentration of plasma in the absence of bacteria, were included in all $\mathrm{CV}$ assays. The $\mathrm{OD}_{595}$ readings of the blanks were subtracted from the $\mathrm{OD}_{595}$ readings of the test 
samples to account for any nonspecific binding of $\mathrm{CV}$ in the wells.

To test the effect of the enzymatic dispersal agents, $S$. aureus overnight cultures were diluted to an $\mathrm{OD}_{600}$ of 0.1 using TSB with $10 \%$ human plasma, seeded into 96-well plates, and incubated statically for 24 hours at $37^{\circ} \mathrm{C}$. The media was removed, and biofilms were washed with PBS. The biofilms were then treated with various concentrations of the dispersal agents for 2 hours or 24 hours at $37^{\circ} \mathrm{C}$, and the $\mathrm{CV}$ assay was performed as described earlier.

\section{Scanning electron microscopy}

Glass coverslips were coated with $1 \mathrm{mg} / \mathrm{mL}$ human fibrinogen in PBS for 24 hours at $4^{\circ} \mathrm{C}$ and then incubated with MRSA SA5120 in TSB and 10\% human plasma for 24 hours. Biofilms were washed, treated with dispersing enzymes for 2 hours, and prepared for scanning electron microscopy (SEM) as previously described. ${ }^{11}$ In brief, biofilms were fixed in $2.5 \%$ phosphate-buffered glutaraldehyde for 1 hour at $4^{\circ} \mathrm{C}$, washed with phosphate buffer, and then dehydrated with serially increasing concentrations of ethanol $(50 \%, 70 \%, 80 \%$, $90 \%, 95 \%$, and $100 \%$ ) in water for 10 minutes each at $4{ }^{\circ} \mathrm{C}$. The samples were further dehydrated twice more with $100 \%$ ethanol for 10 minutes at $4{ }^{\circ} \mathrm{C}$, treated with $50 \%$ ethanol $/ 50 \%$ hexamethyldisilazane for 5 minutes at room temperature, and then treated with $100 \%$ hexamethyldisilazane for 10 minutes at room temperature. Coverslips were placed in a fume hood overnight to remove any remaining liquid, mounted with carbon tape on specimen stubs, and sputter coated with gold using a Hummer 6.2 Sputter Coater (Anatech USA, Union City, CA, USA). Visualization was performed using a SIGMA VP40 scanning electron microscope (Carl Zeiss Meditec AG, Jena, Germany).

\section{Statistics}

Statistical analysis was conducted using GraphPad Prism v6.04 (GraphPad Software, Inc., La Jolla, CA, USA). Oneway analysis of variance and Dunnett's multiple comparison tests were used to compare group means. $P$-values $\leq 0.05$ were considered statistically significant.

\section{Results and discussion Supplementation of TSB media with human plasma enhances $S$. aureus biofilm formation}

In the past decade, numerous studies have been conducted to develop in vitro $S$. aureus biofilm models that better reflect in vivo wound conditions. To accomplish this, various types of growth media have been examined, ${ }^{12,13}$ multiple microbial species have been cultured together, ${ }^{12,14,15}$ and host components have been supplemented to the media..$^{10,12-15}$ One of the unifying aspects of these studies is the addition of plasma or key components of plasma, such as albumin, ${ }^{15}$ to the media to promote cell attachment and enhance $S$. aureus biofilm formation. ${ }^{10,13}$ Thus, in this study, S. aureus biofilm formation was tested using TSB media supplemented with $0 \%-50 \%$ human plasma. Results showed that the addition of as low as $1 \%$ plasma to TSB significantly increased biomass accumulation for five of the six strains cultured for 24 hours and 48 hours (Figure 1A and B). Similar to a recent study, ${ }^{10}$ it was also observed that supplementing the media with $10 \%$ plasma resulted in the highest bioaccumulation relative to cells grown in media alone. Biofilms inoculated with 50\% plasma showed less attachment to the plate as compared to cells supplemented with $10 \%$ plasma and appeared to form gelatinous clots in the media (data not shown). This suggests that the bacteria formed a biofilm island in the media and primarily attached to each other rather than the plate. This is consistent with a recent study involving a Pseudomonas aeruginosa and $S$. aureus dual species biofilm model cultured in $50 \%$ plasma that indicated that $S$. aureus coagulated the plasma to form a "host-derived matrix" to which the bacteria could adhere instead of attaching to artificial surfaces. ${ }^{14}$ When S. aureus biofilms were grown for 48 hours in this study, there were negligible differences in biomass compared to the 24-hour time point (Figure 1B). Based upon these results, a plasma concentration of $10 \%$ and a 24 -hour culture period for biofilm formation were selected for use in all subsequent experiments.

\section{Enzymatic dispersion of S. aureus biofilms over 2 hours}

After establishing the in vitro biofilm model, the ability of dispersal enzymes to degrade mature methicillin-sensitive S. aureus and MRSA biofilms was evaluated. Six strains of S. aureus in vitro biofilms grown over 24 hours were treated with varying concentrations of $\alpha$-amylase, bromelain, lysostaphin, or papain for 2 hours. All strains displayed a dosedependent response to $\alpha$-amylase and bromelain and were markedly sensitive to the three highest papain concentrations (Figure 2). Biofilms were less sensitive to lysostaphin dispersion, where the effect reached a maximum level at $50 \mu \mathrm{g} / \mathrm{mL}$ in all six bacterial strains and did not increase substantially with increasing doses up to $200 \mu \mathrm{g} / \mathrm{mL}$. Maximum levels of biofilm degradation achieved with 
A

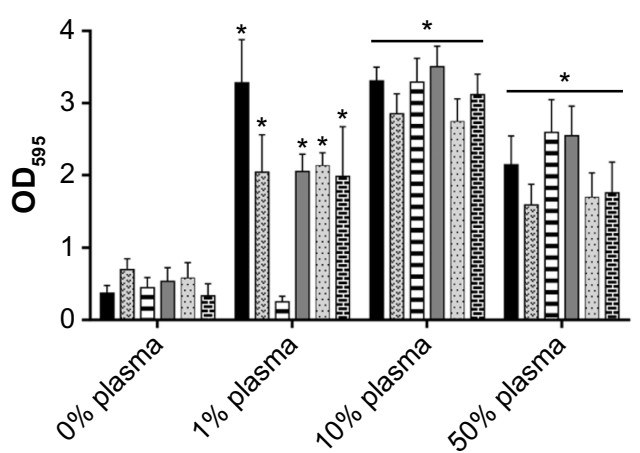

B

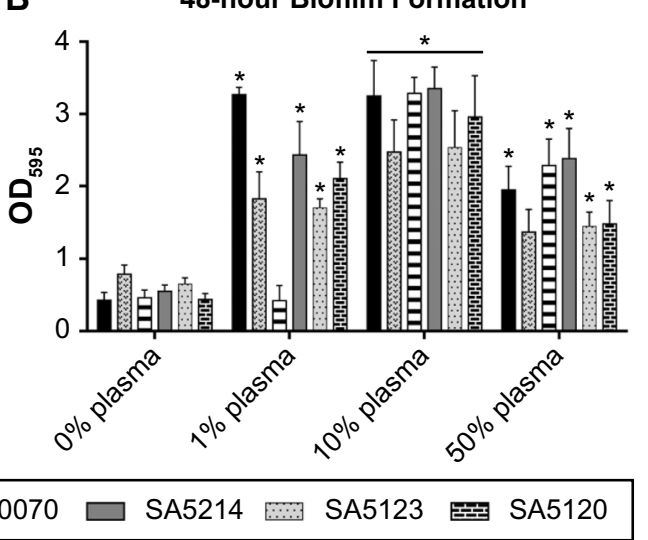

Figure I Effect of human plasma on Staphylococcus aureus biofilm formation.

Notes: S. aureus biofilms were cultured statically for (A) 24 hours or (B) 48 hours in tryptic soy broth supplemented with varying concentrations of human plasma and then quantified using the crystal violet assay. Six strains of $S$. aureus were tested, and values represent the mean + standard deviation of three to four independent experiments with six technical replicates per condition. Data were analyzed using one-way ANOVA followed by Dunnett's multiple comparisons tests. *P $\leq 0.05$ compared to the respective $0 \%$ plasma group.

Abbreviations: OD, optical density; ANOVA, analysis of variance.

lysostaphin in the six strains ranged from $28 \%$ to $72 \%$, whereas maximal reductions in biomass ranged from $94 \%$ to $96 \%, 83 \%$ to $94 \%$, and $85 \%$ to $94 \%$, respectively, for $\alpha$-amylase, bromelain, and papain. The data also show that
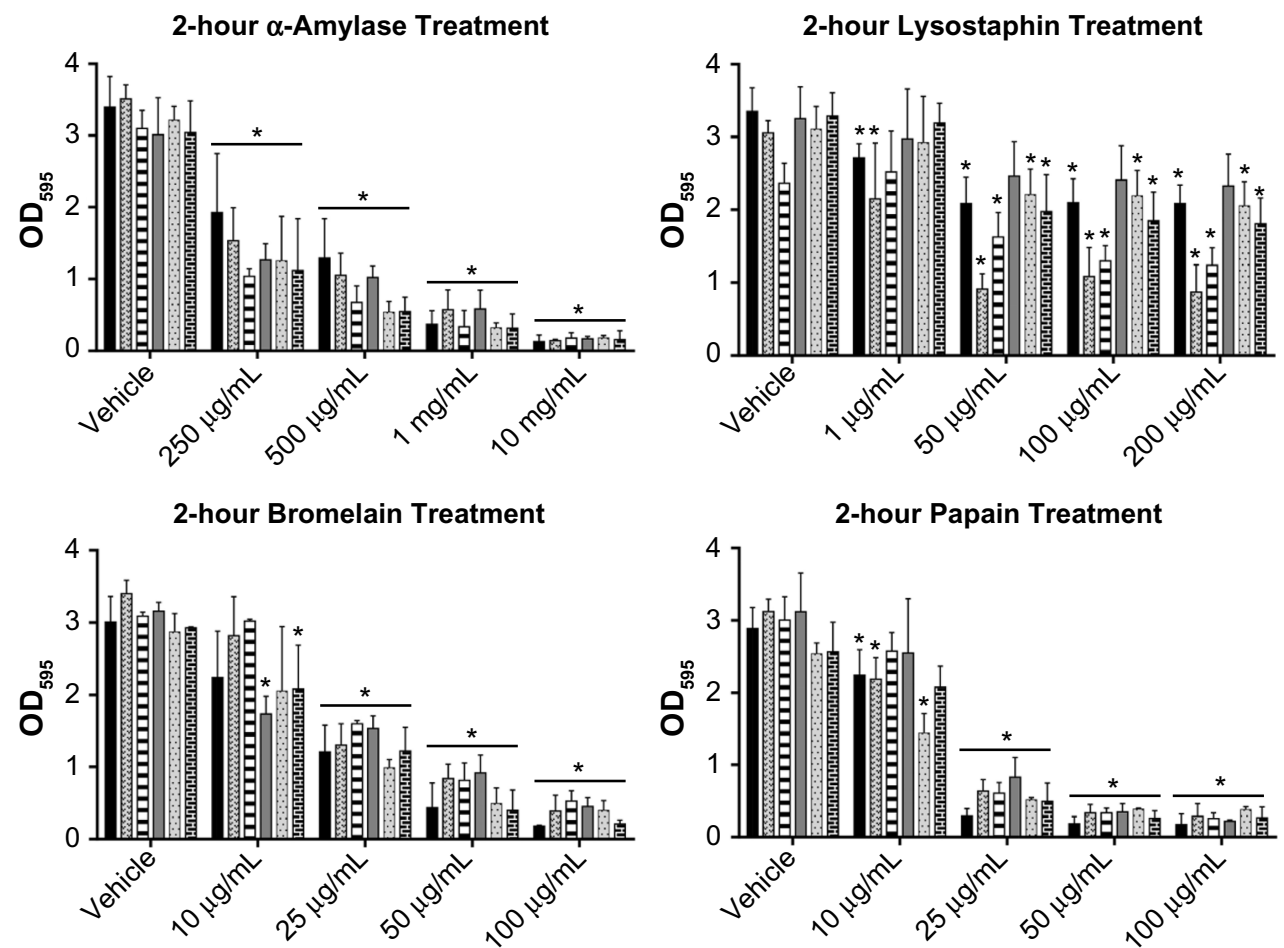

ATCC 29213 ATCC 33591 巳

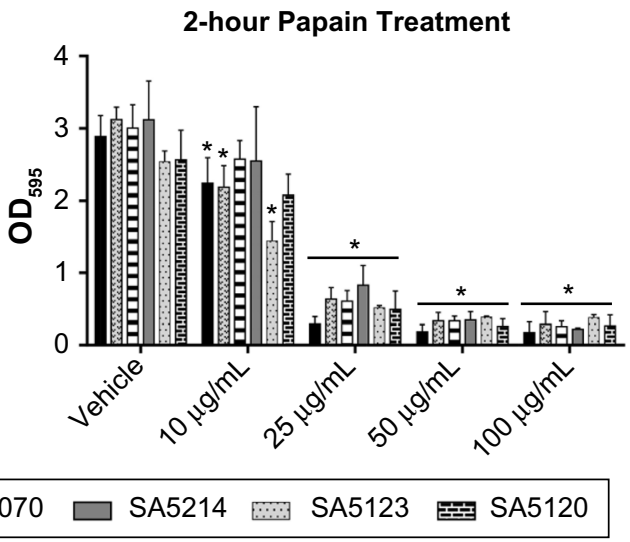

the relative sensitivities of the individual $S$. aureus strains to biofilm degradation were the most variable in response to lysostaphin treatment, indicating strain-dependent sensitivity to dispersal by this agent.

Figure 2 Dispersion of Staphylococcus aureus biofilms with 2-hour enzymatic treatments.

Notes: S. aureus biofilms were cultured for 24 hours in tryptic soy broth with 10\% human plasma and then treated for 2 hours with $\alpha$-amylase, bromelain, lysostaphin, or papain. The crystal violet assay was used to determine the total biomass remaining following the 2-hour treatment. Six strains of $S$. aureus were tested, and values represent the mean + standard deviation of three independent experiments with six technical replicates per condition. Data were analyzed with one-way ANOVA followed by Dunnett's multiple comparisons tests. $* P \leq 0.05$ compared to the respective vehicle control group.

Abbreviation: OD, optical density 
There are limited studies investigating the dispersal of $S$. aureus biofilms using similar exposure times and concentrations for the four enzymatic agents tested in this study. In agreement with our findings, one previous study reported an estimated $86 \%$ dispersion of $S$. aureus biofilms (grown without plasma) after treatment with $10 \mathrm{mg} / \mathrm{mL}$ of $\alpha$-amylase for 3 hours. ${ }^{8}$ However, another study found that a 2-hour treatment of biofilms with $6.25 \mu \mathrm{g} / \mathrm{mL}$ of lysostaphin caused $57 \%$ dispersion, ${ }^{16}$ an effect similar to that observed for two of the strains in this study at higher concentrations of $50-200 \mu \mathrm{g} / \mathrm{mL}$. This disparity may be due to multiple factors, including differences in the biofilm culture conditions and the type of $S$. aureus strains utilized in the two investigations. No previous reports of similar studies using 2-hour exposures of bromelain and papain to disperse $S$. aureus biofilms are available.

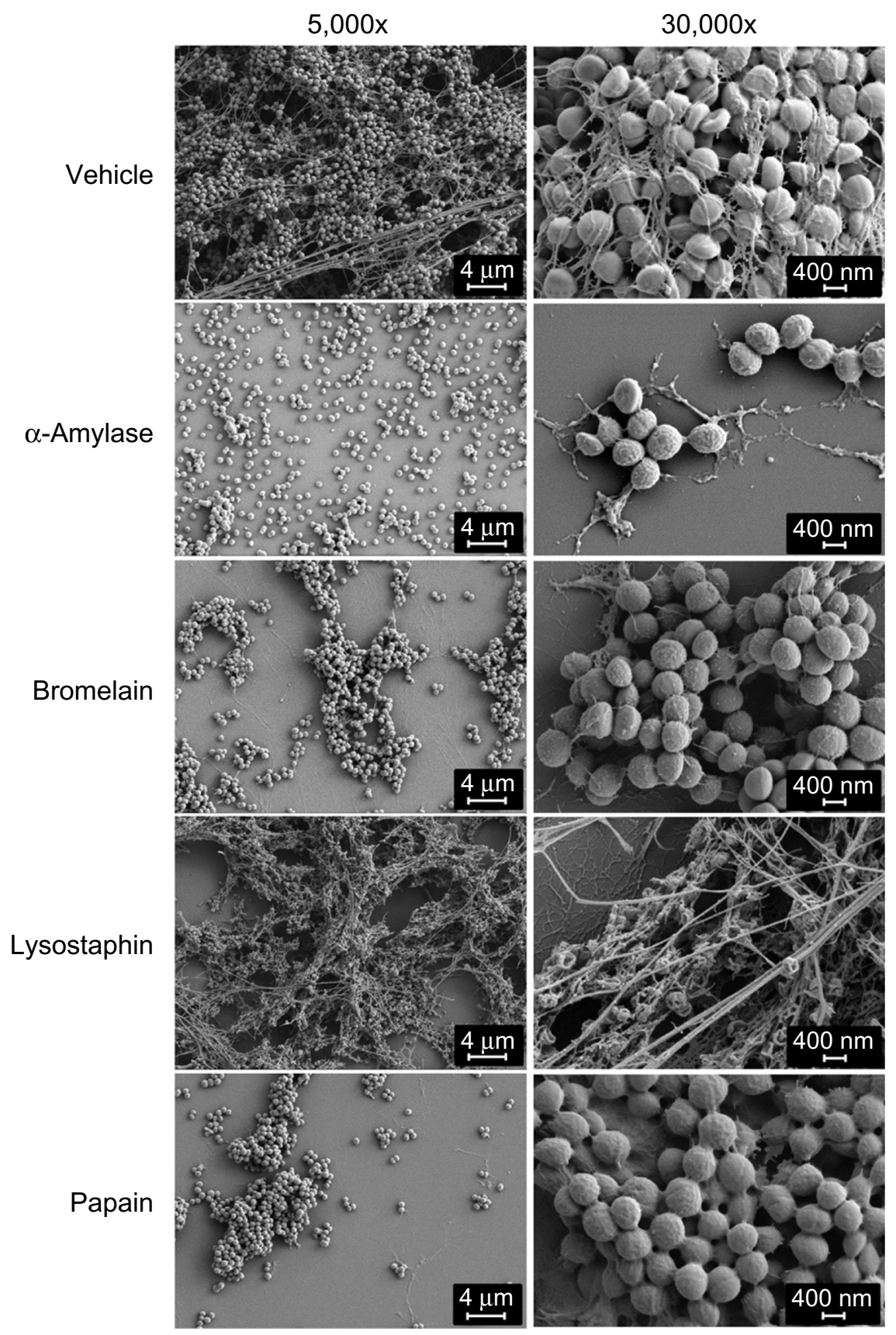

Figure 3 Visualization of dispersal enzyme effect on Staphylococcus aureus biofilms.

Notes: MRSA SA5I20 biofilms were grown for 24 hours on glass coverslips and then treated for 2 hours with vehicle, $\alpha$-amylase $(\mathrm{I} \mathrm{mg} / \mathrm{mL})$, bromelain $(50 \mu g / \mathrm{mL})$, lysostaphin $(200 \mu \mathrm{g} / \mathrm{mL})$, or papain $(50 \mu \mathrm{g} / \mathrm{mL})$. Scanning electron microscopy was used to image the biofilms, and representative images from three independent experiments

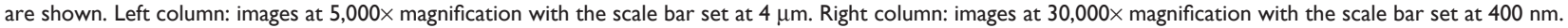
Abbreviation: MRSA, methicillin-resistant S. aureus. 
Visualization of biofilms with SEM at 5,000× showed that, consistent with the $\mathrm{CV}$ assay results, $\alpha$-amylase, bromelain, and papain caused greater degradation of the EPS matrix and bacterial detachment than lysostaphin (left column in Figure 3). Imaging with SEM at 30,000× revealed that lysostaphin altered the cell morphology of S. aureus without much effect on the EPS matrix, which remained largely intact, and that the other three agents did not appear to significantly affect bacterial morphology (right column in Figure 3). These results are consistent with previous findings where treatment with up to $200 \mathrm{mg} / \mathrm{mL}$ of $\alpha$-amylase over 24 hours had no bactericidal effect against planktonic $S$. aureus. ${ }^{8}$ The data are also in agreement with the known mechanism of action of lysostaphin, a well-characterized bactericidal agent that targets the cross-linking pentaglycine bridges of the S. aureus cell wall and can lyse planktonic cells within 10 minutes at concentrations as low as $2-5 \mu \mathrm{g} / \mathrm{mL} .{ }^{17}$ Though the bactericidal activities of bromelain and papain against $S$. aureus have not been clearly elucidated, papain showed no antibacterial effect on 138 isolates of Staphylococcus epidermidis and Staphylococcus haemolyticus after 24 hours of treatment. ${ }^{18}$
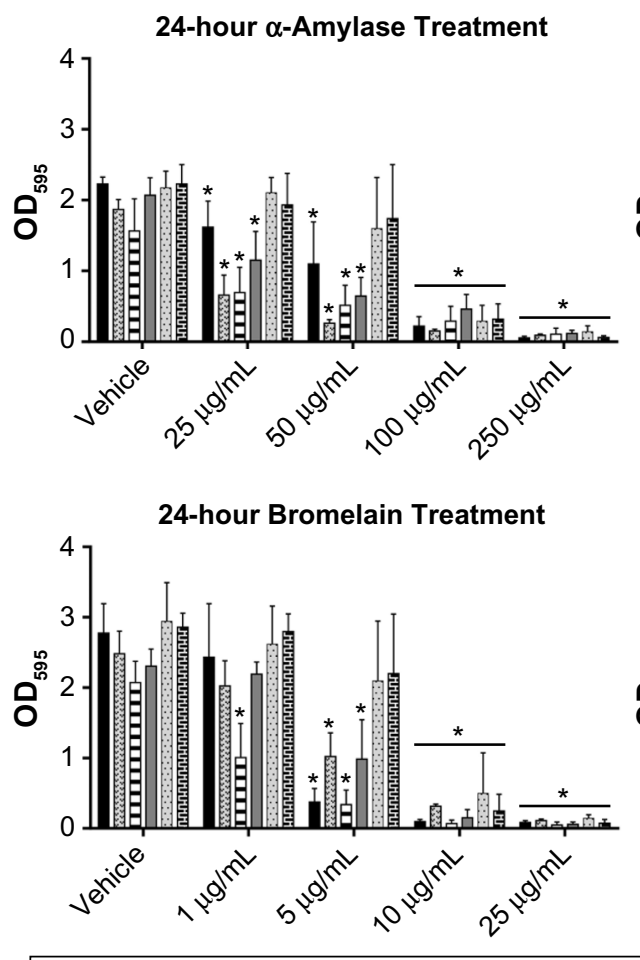

ATCC 29213 ATCC $33591 \quad \sqsupseteq$ IQ007

Figure 4 Degradation of Staphylococcus aureus biofilms after 24-hour treatment with dispersal agents.

Notes: Biofilms were cultured for 24 hours in tryptic soy broth with 10\% human plasma and then treated with $\alpha$-amylase, lysostaphin, bromelain, or papain for 24 hours. The crystal violet assay was used to determine the total biomass remaining after the 24 -hour treatment. Six strains of $S$. aureus were tested, and values represent the mean + standard deviation of four independent experiments with six technical replicates per condition. Data were analyzed with one-way ANOVA followed by Dunnett's multiple comparisons tests. $* P \leq 0.05$ compared to the respective vehicle control group.

Abbreviations: OD, optical density; ANOVA, analysis of variance.

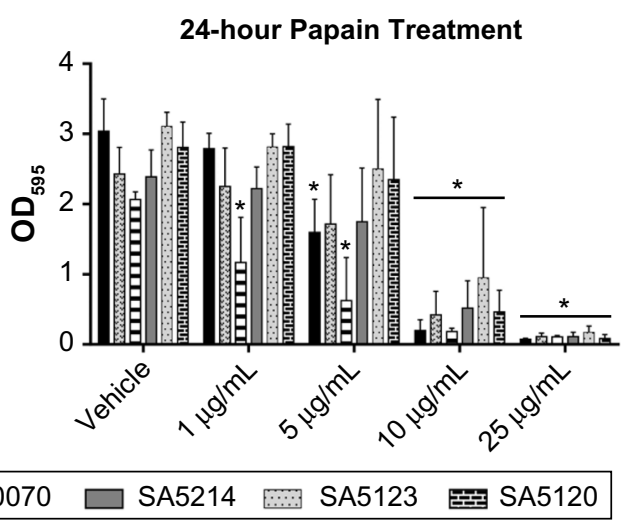

\section{Enzymatic dispersion of S. aureus biofilms over 24 hours}

After establishing the enzymatic activities of the four agents for 2-hour treatments, biofilm degradation was assessed using a longer duration of treatment and reduced dispersal agent concentrations. S. aureus biofilms were cultured for 24 hours with $10 \%$ plasma and then treated for 24 hours with $\alpha$-amylase, lysostaphin, bromelain, or papain (Figure 4). The highest enzyme concentrations achieved the following decreases in biofilm biomass in the six bacterial strains: $92 \%-97 \%$ for $\alpha$-amylase, $95 \%-98 \%$ for bromelain, and $94 \%-98 \%$ for papain. Thus, increasing the length of enzymatic treatment for these three agents allowed reduction of the enzyme concentration required to effectively degrade biofilms. For lysostaphin, the greatest reduction in biofilm biomass for all six bacterial strains was achieved at $50 \mu \mathrm{g} / \mathrm{mL}$ and ranged from $49 \%$ to $76 \%$. The highest dose of lysostaphin, that is, $250 \mu \mathrm{g} / \mathrm{mL}$, was less effective at dispersing some of the MRSA strains and caused only $6 \%-35 \%$ reduction in biomass. The reason for this pattern of effect in lysostaphin-treated cultures is unknown, though one possible

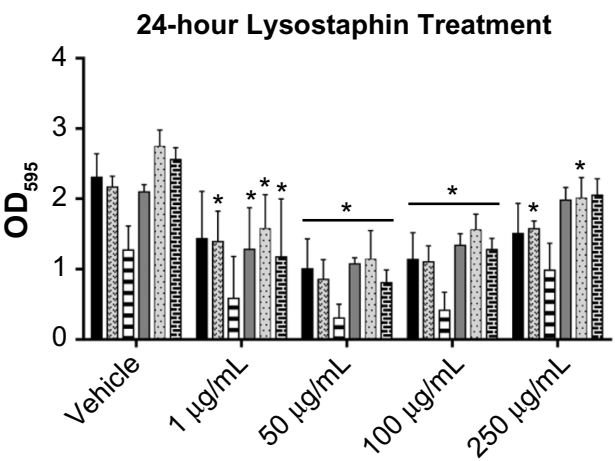


explanation is that the highest dose caused a more rapid lysis of bacteria and release of inhibitory cellular components resulting in diminished enzymatic activity. ${ }^{19}$

Several previous studies involving 24-hour treatment of $S$. aureus biofilms (cultured without plasma) with the four enzymes used in the current investigation have been reported. For example, Wu et $\mathrm{al}^{16}$ showed that $6.25 \mu \mathrm{g} / \mathrm{mL}$ of lysostaphin was needed to cause a sevenfold reduction in $S$. aureus biomass. In contrast, only a one- to twofold decrease in biomass for most of the $S$. aureus strains at 8-16 times that concentration was observed in this study. Sugimoto et $\mathrm{al}^{20}$ reported that $S$. aureus biofilms were completely dispersed using $5 \mu \mathrm{g} / \mathrm{mL}$ of papain, which is a two- to fivefold lower concentration than that required to thoroughly disrupt biofilms in the current investigation. A previous study of $\alpha$-amylase applied to $S$. aureus biofilms for 24 hours showed that $20-100 \mathrm{mg} / \mathrm{mL}$ was required to achieve maximal dispersion, ${ }^{8}$ whereas concentrations of only $100-250 \mu \mathrm{g} / \mathrm{mL}$ caused complete dispersion in our study. Disparities in the results from this and previous studies could be due to differences in key study parameters, such as the enzyme source and purity, enzyme vehicle, strains of $S$. aureus, and composition of the biofilm growth medium. At this time, no relevant previous studies have been conducted concerning the impact of a 24-hour treatment with bromelain on $S$. aureus biofilms.

\section{Conclusion}

This study focused on examining the in vitro activity of dispersal enzymes, including two clinically used enzymatic debridement agents (papain and bromelain), against biofilms formed by clinical wound isolates cultured using human plasma. Overall, we demonstrated that addition of human plasma to growth media enhanced in vitro $S$. aureus biofilm formation and that the clinical MRSA strains required higher plasma concentrations for maximal biofilm formation compared to the antibiotic-sensitive laboratory strain (ATCC 29213). Utilizing the human plasma biofilm model, four dispersing enzymes ( $\alpha$-amylase, lysostaphin, bromelain, and papain) were tested on $S$. aureus biofilms at 2 hours and 24 hours, and optimal treatment concentrations were determined. Compared to prior studies of biofilms cultured without plasma, our findings suggest that biofilms established in growth media supplemented with $10 \%$ human plasma may be more recalcitrant to dispersal by lysostaphin and papain. It should be noted that because human plasma significantly increases biofilm biomass, it is difficult to directly compare our data to the efficacy of dispersing enzymes on biofilms cultured with little or no plasma. The decreased dispersal observed in this study may simply be due to the presence of more substrate for the enzyme to break down. However, human plasma can upregulate expression of cell surface molecules involved in bacterial adhesion to surfaces, increase cell wall thickness, and change the composition of the extracellular matrix of S. aureus biofilms, ${ }^{10}$ all of which could influence susceptibility of biofilms to enzymatic dispersal. Future studies will focus on examining the effects of human plasma on biofilm formation and dispersal enzyme activity in other commonly isolated wound pathogens.

\section{Acknowledgments}

This project was supported by the Naval Medical Research Unit San Antonio work unit number G1025. CW was supported, in part, by an appointment to the Research Participation Program at the Naval Medical Research Unit San Antonio, administered by ORAU through the US Department of Energy Oak Ridge Institute for Science and Education. MRSA IQ0070 was provided by Dr Kai Leung (US Army Dental Trauma and Research Detachment, JBSA-Fort Sam Houston, TX, USA). MRSA SA5214, SA5123, and SA5120 were obtained from a repository at the US Army Institute for Surgical Research. The authors thank Dr Tao You (US Army Dental and Trauma Research Detachment) for assistance with SEM. The views expressed in this article are those of the authors and do not necessarily reflect the official policy or position of the Department of the Navy, Department of Defense, or the US Government. Authors are employees or contractors of the US Government. This work was prepared as part of their official duties. Title 17 USC $\S 105$ provides that "Copyright protection under this title is not available for any work of the US Government." Title 17 USC $\$ 101$ defines a US Government work as a work prepared by a military service member or employee of the US Government as part of that person's official duties.

\section{Disclosure}

The authors report no conflicts of interest in this work.

\section{References}

1. Donlan RM, Costerton JW. Biofilms: survival mechanisms of clinically relevant microorganisms. Clin Microbiol Rev. 2002;15(2):167-193.

2. Thallinger B, Prasetyo EN, Nyanhongo GS, Guebitz GM. Antimicrobial enzymes: an emerging strategy to fight microbes and microbial biofilms. Biotechnol J. 2013;8(1):97-109. 
3. de la Fuente-Nunez C, Reffuveille F, Mansour SC, et al. D-enantiomeric peptides that eradicate wild-type and multidrug-resistant biofilms and protect against lethal Pseudomonas aeruginosa infections. Chem Biol. 2015;22(2):196-205.

4. Conlon BP, Nakayasu ES, Fleck LE, et al. Activated ClpP kills persisters and eradicates a chronic biofilm infection. Nature. 2013; 503(7476):365-370.

5. Davis S, Nusbaum A. What is new in debridement? In: Sen C, editor. Advances in Wound Care. Vol. 1. New York: Mary Ann Liebert; 2010: 535-541.

6. Bolton L, Fattu AJ. Topical agents and wound healing. Clin Dermatol. 1994;12(1):95-120.

7. Di Poto A, Sbarra MS, Provenza G, Visai L, Speziale P. The effect of photodynamic treatment combined with antibiotic action or host defence mechanisms on Staphylococcus aureus biofilms. Biomaterials. 2009;30(18):3158-3166.

8. Craigen B, Dashiff A, Kadouri DE. The use of commercially available alpha-amylase compounds to inhibit and remove Staphylococcus aureus biofilms. Open Microbiol J. 2011;5:21-31.

9. Pettit RK, Weber CA, Pettit GR. Application of a high throughput Alamar blue biofilm susceptibility assay to Staphylococcus aureus biofilms. Ann Clin Microbiol Antimicrob. 2009;8:28.

10. Cardile AP, Sanchez CJ Jr, Samberg ME, et al. Human plasma enhances the expression of Staphylococcal microbial surface components recognizing adhesive matrix molecules promoting biofilm formation and increases antimicrobial tolerance in vitro. BMC Res Notes. 2014;7:457.

11. Araujo JC, Teran FC, Oliveira RA, et al. Comparison of hexamethyldisilazane and critical point drying treatments for SEM analysis of anaerobic biofilms and granular sludge. J Electron Microsc (Tokyo). 2003;52(4):429-433.

12. Sun Y, Dowd SE, Smith E, Rhoads DD, Wolcott RD. In vitro multispecies Lubbock chronic wound biofilm model. Wound Repair Regen. 2008;16(6):805-813.
13. Chen P, Abercrombie JJ, Jeffrey NR, Leung KP. An improved medium for growing Staphylococcus aureus biofilm. J Microbiol Methods. 2012;90(2):115-118.

14. DeLeon S, Clinton A, Fowler H, Everett J, Horswill AR, Rumbaugh KP. Synergistic interactions of Pseudomonas aeruginosa and Staphylococcus aureus in an in vitro wound model. Infect Immun. 2014; 82(11):4718-4728

15. Kart D, Tavernier S, Van Acker H, Nelis HJ, Coenye T. Activity of disinfectants against multispecies biofilms formed by Staphylococcus aureus, Candida albicans and Pseudomonas aeruginosa. Biofouling. 2014;30(3):377-383.

16. Wu JA, Kusuma C, Mond JJ, Kokai-Kun JF. Lysostaphin disrupts Staphylococcus aureus and Staphylococcus epidermidis biofilms on artificial surfaces. Antimicrob Agents Chemother. 2003;47(11): 3407-3414.

17. Kusuma CM, Kokai-Kun JF. Comparison of four methods for determining lysostaphin susceptibility of various strains of Staphylococcus aureus. Antimicrob Agents Chemother. 2005;49(8):3256-3263.

18. Oliveira HLCD, Fleming MECK, Silva PV, et al. Influence of papain in biofilm formed by methicillin-resistant Staphylococcus epidermidis and methicillin-resistant Staphylococcus haemolyticus isolates. Braz J Pharm Sci. 2014;50(2):261-267.

19. Schaffner W, Melly MA, Hash JH, Koenig MG. Lysostaphin: an enzymatic approach to staphylococcal disease. I. In vitro studies. Yale J Biol Med. 1967;39(4):215-229.

20. Sugimoto S, Iwamoto T, Takada K, et al. Staphylococcus epidermidis Esp degrades specific proteins associated with Staphylococcus aureus biofilm formation and host-pathogen interaction. J Bacteriol. 2013;195(8):1645-1655.
Infection and Drug Resistance

\section{Publish your work in this journal}

Infection and Drug Resistance is an international, peer-reviewed openaccess journal that focuses on the optimal treatment of infection (bacterial, fungal and viral) and the development and institution of preventive strategies to minimize the development and spread of resistance. The journal is specifically concerned with the epidemiology of antibiotic

\section{Dovepress}

resistance and the mechanisms of resistance development and diffusion in both hospitals and the community. The manuscript management system is completely online and includes a very quick and fair peerreview system, which is all easy to use. Visit http://www.dovepress.com/ testimonials.php to read real quotes from published authors. 